\title{
CHARACTERISATION OF THE PSI WHOLE BODY COUNTER BY RADIOGRAPHIC IMAGING
}

\author{
S. Mayer ${ }^{1, *}$, M. Boschung ${ }^{1}$, K. Meier ${ }^{1}$, J.-P. Laedermann ${ }^{2}$ and F. O. Bochud ${ }^{2}$ \\ ${ }^{1}$ Paul Scherrer Institut (PSI), Radiation Metrology Section, CH-5232 Villigen PSI, Switzerland \\ ${ }^{2}$ Institute of Radiation Physics (IRA), University Hospital Center and University of Lausanne, \\ Rue du Grand-Pré 1, CH-1007 Lausanne, Switzerland \\ *Corresponding author: sabine.mayer@psi.ch
}

\begin{abstract}
A joint project between the Paul Scherrer Institut (PSI) and the Institute of Radiation Physics was initiated to characterise the PSI whole body counter in detail through measurements and Monte Carlo simulation. Accurate knowledge of the detector geometry is essential for reliable simulations of human body phantoms filled with known activity concentrations. Unfortunately, the technical drawings provided by the manufacturer are often not detailed enough and sometimes the specifications do not agree with the actual set-up. Therefore, the exact detector geometry and the position of the detector crystal inside the housing were determined through radiographic images. X-rays were used to analyse the structure of the detector, and ${ }^{60} \mathrm{Co}$ radiography was employed to measure the core of the germanium crystal. Moreover, the precise axial alignment of the detector within its housing was determined through a series of radiographic images with different incident angles. The hence obtained information enables us to optimise the Monte Carlo geometry model and to perform much more accurate and reliable simulations.
\end{abstract}

\section{INTRODUCTION}

The Paul Scherrer Institut (PSI) runs a whole body counter based on a p-type high-purity germanium (HPGe) coaxial detector mounted above a canvas chair in a shielded room. The detector is used to measure radionuclides in the human body that emit photons with energies between $50 \mathrm{keV}$ and $2 \mathrm{MeV}$.

In a joint project between the PSI and the Institute of Radiation Physics, the whole body counter is characterised in detail through measurements and simulations. The extensive project includes the following stages: first, the detector geometry is measured accurately through radiographic imaging. Precise knowledge of the detector geometry is crucial for all subsequent simulations where complex human body phantoms filled with known activity concentrations are studied. However, to make sure that the germanium detector with its alignment in the housing is properly simulated, measurements with point sources and simplified geometries are done in the next stage. Once the detector model has been verified for these base cases, more complicated phantom set-ups filled with different radionuclides can be assembled. In this phase, the spectra and efficiencies for different radionuclides will be determined for the calibration phantom, where different body sizes are represented by different standardised configurations of polyethylene bricks, in which small tubes of calibration sources can be introduced. Finally, the simulation can be extended to voxel phantoms, which approximate the human body and its organs best.
The general approach to the characterisation of HPGe detectors to obtain a good model for use in Monte Carlo simulation has been described in the literature before ${ }^{(1-4)}$. However, in this paper, the first stage of the project is described in detail, since the use of imaging plates (IP) in characterising HPGe detectors is a new approach. With the help of radiography analysis, precise measurements of the inner detector geometry could be performed for the PSI whole body counter. Since the technical drawings provided by the manufacturer are often not detailed enough and sometimes the specifications do not agree with the actual set-up, the use of radiographic images essentially improves the first step of the assessment of the detector geometry by providing a much better, and also time saving, starting point for the iterative development of the detector model.

\section{MATERIALS AND METHODS}

\section{Whole body counter}

The PSI whole body counter ${ }^{(5)}$ is based on a p-type HPGe coaxial detector manufactured by Canberra (former: Eurysis Mesures, type: EGPC 100-225-R). According to the manufacturer, the cylindrical germanium crystal has a diameter of $80.4 \mathrm{~mm}$ and a length of $83.8 \mathrm{~mm}$.

\section{Imaging plates}

The imaging was done on IP of Fujifilm, which are reusable two-dimensional sensors for the detection 
and storage of ionising radiation energy in photostimulable phosphor crystals. The Fujifilm Bio-imaging scanner BAS-2500 was used to read the IP.

\section{Radiographic imaging}

Two different radiographic imaging methods were used. A photon spectrum from a standard X-ray machine was used to reproduce the structure of the detector as well as a ${ }^{60} \mathrm{Co}$ radiography to penetrate deeper in the crystal due to the higher photon energy and measure the core of the germanium crystal (Figure 1). During the radiography, the detector was
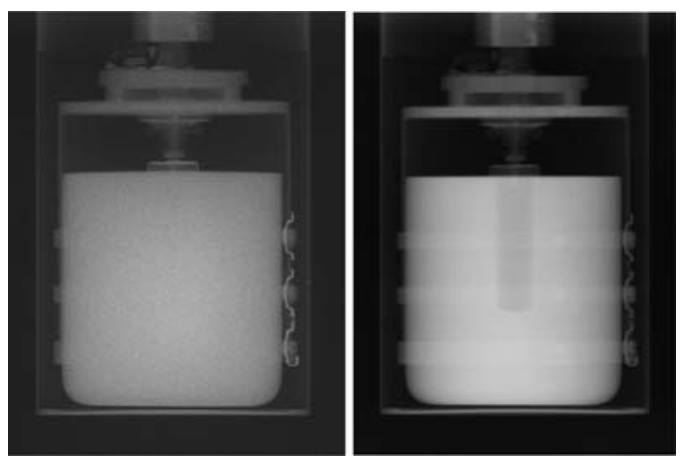

Figure 1. Radiographic image after X-ray (left) and ${ }^{60} \mathrm{Co}$ exposure (right). positioned in front of the IP, which were mounted a distance of $5.2 \mathrm{~m}$ from the radiation source. Because of the large distance between the source and the IP, the optical distortion on the IP could be neglected. In order to obtain information about the axial alignment of the detector in the detector housing, the radiography was carried out not only in one incident direction, but with different incident angles. The selected incident angles were $0^{\circ}, 45^{\circ}, 90^{\circ}, 135^{\circ}$ and $180^{\circ}$. The exposure time for the $\mathrm{X}$-ray imaging with a radiation quality of N-300 was $10 \mathrm{~min}$, corresponding to a dose of $0.4 \mathrm{mGy}$. The ${ }^{60} \mathrm{Co}$ irradiations were performed for an exposure time of $15 \mathrm{~min}$, corresponding to a dose of $50 \mathrm{mGy}$, which led to an overexposure of the IP. However, it was found that re-reading the IP with the scanner several times without erasing the image made the core of the detector crystal visible and thus its dimensions could be measured.

\section{RESULTS}

The dimensions on the images have been measured with the image processing program Corel Draw. For each image, the diameter of the detector housing of $100 \mathrm{~mm}$ was taken as reference, and all other dimensions were normalised to this distance. In Figure 2, a radiographic image of the germanium detector and a detailed drawing of the detector on the basis of the obtained information are shown. In Table 1, the mean values of the measured dimensions corresponding to the specifications in Figure 2
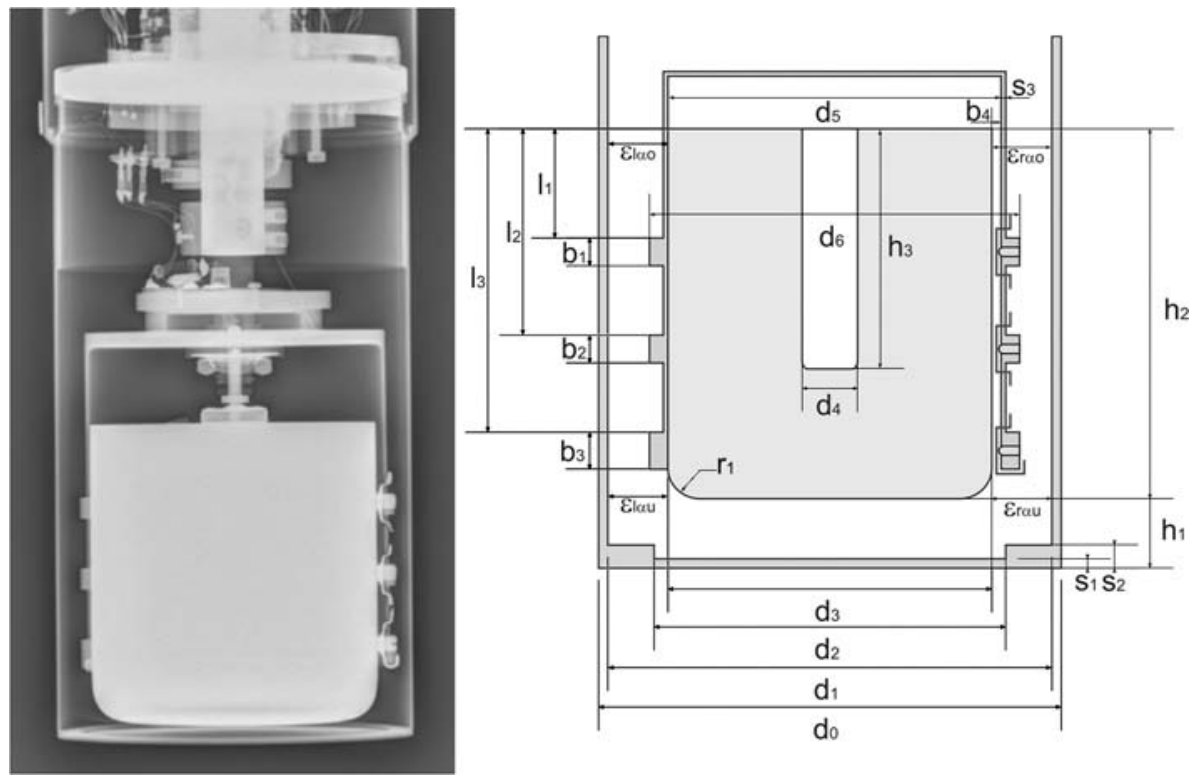

Figure 2. Radiography of the germanium detector and detailed drawing of the detector on the basis of radiographic images. 


\section{S. MAYER ET AL.}

Table 1. Comparison of detector dimensions provided by the manufacturer and determined by radiographic imaging.

\begin{tabular}{|c|c|c|}
\hline Detector detail & $\begin{array}{c}\text { Manufacturer } \\
\text { specification }(\mathrm{mm})\end{array}$ & $\begin{array}{l}\text { This work } \\
\text { (mm) }\end{array}$ \\
\hline $\mathrm{d} 0$ & 100 & Reference \\
\hline $\mathrm{d} 1$ & $-{ }^{\mathrm{a}}$ & 98.1 \\
\hline $\mathrm{d} 2$ & $-{ }^{\mathrm{a}}$ & 87.0 \\
\hline $\mathrm{d} 3$ & 80.4 & 80.4 \\
\hline $\mathrm{d} 4$ & 14.0 & 13.7 \\
\hline d5 & $-{ }^{\mathrm{a}}$ & 82.4 \\
\hline d6 & 87.8 & $-{ }^{\mathrm{a}}$ \\
\hline h1 & 3 & 3.1 \\
\hline h2 & 83.8 & 84.8 \\
\hline h3 & 50.0 & 49.8 \\
\hline s1 & 1 & 1.2 \\
\hline s2 & $-{ }^{\mathrm{a}}$ & 2.1 \\
\hline s3 & $-^{\mathrm{a}}$ & 1.0 \\
\hline $\mathrm{r} 1$ & $-{ }^{\mathrm{a}}$ & 8.2 \\
\hline b1 & $-{ }^{\mathrm{a}}$ & 6.2 \\
\hline b2 & $-^{\mathrm{a}}$ & 6.2 \\
\hline b3 & $-{ }^{\mathrm{a}}$ & 8.4 \\
\hline 11 & $-^{\mathrm{a}}$ & 20.4 \\
\hline 12 & $-{ }^{\mathrm{a}}$ & 40.6 \\
\hline 13 & $-{ }^{\mathrm{a}}$ & 60.9 \\
\hline $\begin{array}{l}\text { Detector volume } \\
\left(\mathrm{cm}^{3}\right)\end{array}$ & 413 & 418 \\
\hline
\end{tabular}

In the case of the measured data, the results are mean values over $2-6$ images.

${ }^{\mathrm{a}}$ No information.

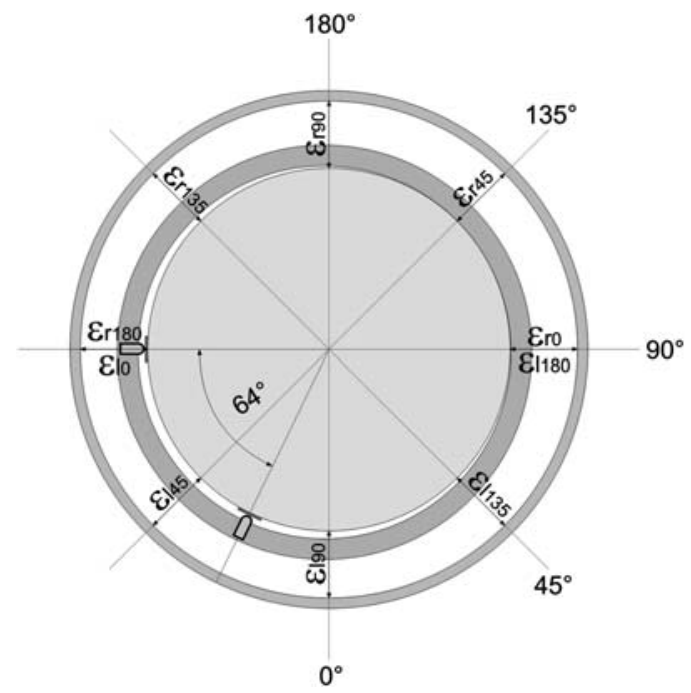

Figure 3. Axial alignment of the detector.

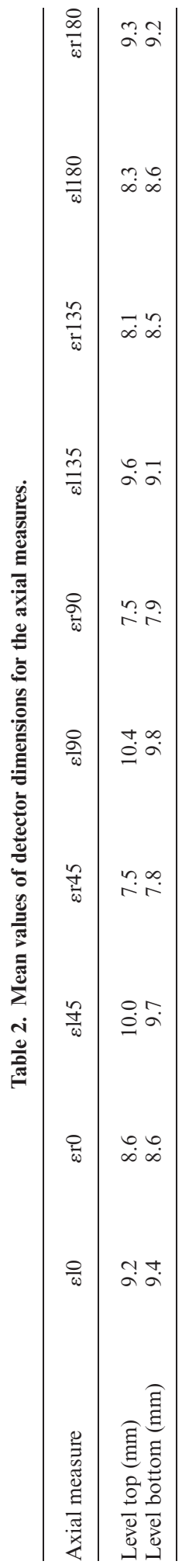


are compared with the manufacturer's specifications. The mean values are averaged over the results of radiographic images taken from different incident angles. The standard uncertainties of the mean values are $0.2-3.5 \%$ for the detector details $\mathrm{d} 0-\mathrm{d} 6$, $\mathrm{h} 1-\mathrm{h} 3$ and $11-13$. For the detector details $\mathrm{s} 1-\mathrm{s} 3, \mathrm{r} 1$ and $\mathrm{b} 1-\mathrm{b} 3$, the standard uncertainty was estimated to be $2-10 \%$. On the basis of the radiographic measurements, the volume of the detector is 418 $\mathrm{cm}^{3}$ as opposed to a volume of $413 \mathrm{~cm}^{3}$ as stated by the manufacturer. In Figure 3, the axial alignment of the crystal in the detector housing is shown. The corresponding results for the geometrical dimensions are listed in Table 2.

\section{CONCLUSION}

The exact detector geometry and the detector crystal positioning inside the housing were determined by means of radiographic images. In fact, details were found by this method, which were previously unknown. The gained information enables an optimisation of the Monte Carlo model for the investigated detector and forms a basis for more accurate and reliable simulations.

\section{FUNDING}

This work was partly supported by the Swiss Federal Nuclear Safety Inspectorate (ENSI, contract $\mathrm{N}^{\circ} 100694$ ).

\section{REFERENCES}

1. Vargas, M. J., Timón, A. F., Díaz, N. C. and Sánchez, D. P. Influence of the geometrical characteristics of an $\mathrm{HpGe}$ detector on its efficiency. J. Radioanal. Nucl. Chem. 253(3), 439-443 (2002).

2. Dryak, P. and Kovar, P. Experimental and MC determination of HPGe detector efficiency in the 40-2754 keV energy range for measuring point source geometry with the source-to-detector distance of $25 \mathrm{~cm}$. Appl. Radiat. Isot. 64, 1346-1349 (2006).

3. Bochud, F. O., Bailat, C., Buchillier, T., Byrde, F., Schmid, E. and Laedermann, J.-P. Simple Monte-Carlo method to calibrate well-type HPGe detector. Nucl. Instrum. Methods Phys. Res. A. 569, 790-795 (2006).

4. Schläger, M. Precise modelling of coaxial germanium detectors in preparation for a mathematical calibration. Nucl. Instrum. Methods Phys. Res. A. 580, 137-140 (2007).

5. Boschung, M. The high purity germanium detector wholebody monitor at PSI. Radiat. Prot. Dosim. 79(1-4), 481-484 (1998). 